\title{
Entwicklung eines Geometrienormals zur Kalibrierung von pneumatischen Abstandssensoren
}

\author{
Matthias Eifler, Patrick Schäfer, Jörg Seewig \\ Lehrstuhl für Messtechnik \& Sensorik, TU Kaiserslautern, Kaiserslautern \\ Tel.: 0631-2054120, e-mail: meifler@mv.uni-kl.de
}

\section{Kurzfassung}

Die pneumatische Messtechnik ist robust gegen Schmutz und Kühlmittel und daher gut für die Inline-Messtechnik geeignet. Viele physikalische Grundlagen des Verfahrens sind unzureichend beschrieben, können aber dank moderner Rechnersysteme mit neuen Möglichkeiten untersucht werden. Dazu gehören auch Kalibrierstrategien, deren Untersuchungen vorgestellt werden. Im Speziellen werden die Entwicklung von zwei Geometrienormalen, Messergebnisse und zwei Ansätze zur virtuellen Abtastung der Normale gezeigt. Zwei Kalibrierstrategien werden präsentiert und die Ergebnisse verschiedener Abtastungen miteinander verglichen.

\section{Einleitung}

Momentan werden pneumatische Sensoren relativ kalibriert. Probleme wie die elastische Verformung der Messeinrichtung durch den Staudruck der angeblasenen Oberfläche bleiben unberücksichtigt und können zu unerwarteten Abweichungen führen. Zur Verbesserung der Rückführbarkeit [1] wird ein Ansatz zur absoluten Kalibrierung vorgestellt, wobei die Nulllage geometrisch und eindeutig in gewissen Grenzen rückführbar ermittelt wird.

\section{Pneumatische Sensoren - Aufbau und Modellierung}

Der Abstand zwischen Werkstück und Sensor wird in der pneumatischen Messtechnik durch Druck- oder Geschwindigkeitsänderung im Strömungskanal des 
Sensors ermittelt [2]. Dabei wird der Effekt genutzt, dass eine Strömung durch ihren engsten Querschnitt bestimmt wird. Eine Abstandsänderung muss zu einer Variation dieses engsten Querschnitts führen, um messbar zu sein [2]. Vorteil ist die prozessintegrierte Reinigung, was das Verfahren für raue Messumgebungen und die Inline-Messtechnik qualifiziert [3].

Der Aufbau pneumatischer Sensoren ist in Bild 1 dargestellt. Gefilterte Luft, die auf einen konstanten Vordruck $p_{V}$ geregelt wird, strömt durch eine Vordüse mit dem Querschnitt $A_{V}$. Der Druck fällt dabei auf den Messdruck $p_{M}$ ab. Dieser wird unter anderem durch den engsten Querschnitt zwischen Messdüse und Werkstück $A_{M}$ bestimmt. Der Abstand $s$ zwischen diesen Komponenten kann eindeutig einem Druckverhältnis $p_{M} / p_{V}$ zugeordnet werden.

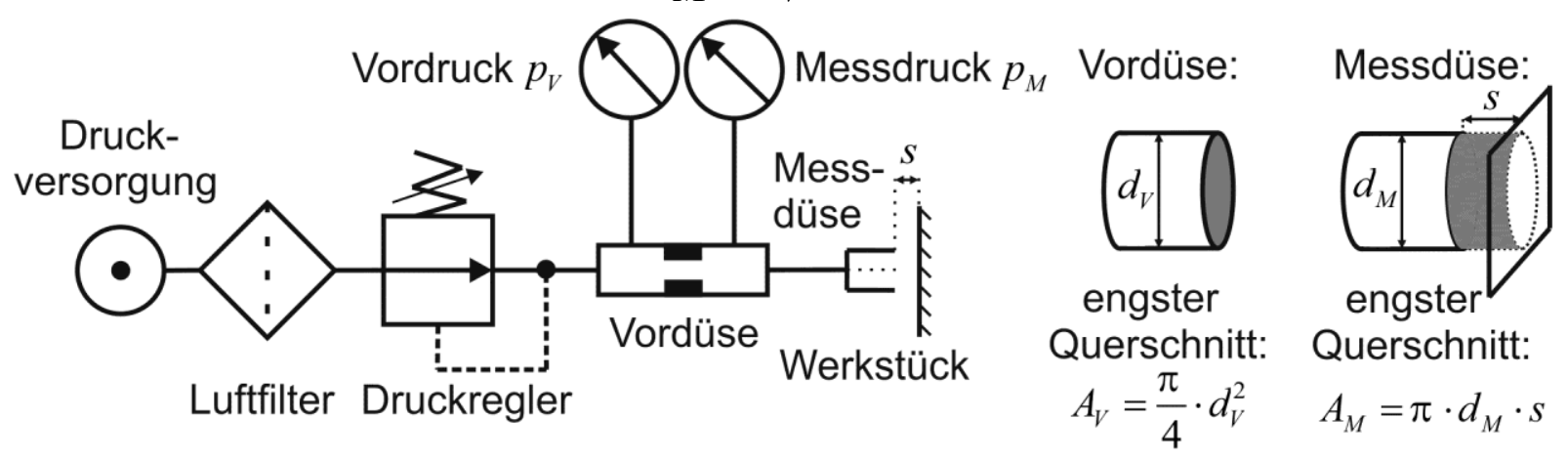

Bild 1: Aufbau pneumatischer Abstandssensoren

Die Kennlinie des Sensors ist eine Funktion der Querschnitte $A_{M}, A_{V}$, des Adiabatenkoeffizienten $\kappa$ des eingesetzten Arbeitsmediums sowie der Druckverhältnisse $\omega_{U}=p_{U} / p_{M}, \omega_{M}=p_{M} / p_{V}$ wobei $p_{U}$ den Umgebungsdruck beschreibt [4]. Für den praktischen Einsatz werden die Vordüse im Unterschall und die Messdüse im Überschall betrieben. Wird dieser Strömungsfall erreicht, so wird der Faktor $\omega_{U}$ durch das kritische Druckverhältnis $\beta$ beschrieben. Der Faktor $\omega_{M}$ ist vom Druckgefälle über die Vordüse abhängig:

$$
\omega_{U}=\beta ; \omega_{M}=p_{M} / p_{V}
$$

Die Sensor-Kennlinie kann dann mathematisch wie folgt beschrieben werden [4]:

$$
s=\frac{d_{V}^{2} \sqrt{\left(\omega_{M}^{2 / \kappa}-\omega_{M}^{(\kappa+1) / \kappa}\right)}}{4 \cdot d_{M} \sqrt{\left(\omega_{U}^{2 / \kappa}-\omega_{U}^{(\kappa+1) / \kappa}\right)}}
$$


Eine beispielhafte Kennlinie in Abhängigkeit des Druckverhältnisses $\omega_{M}=p_{M} / p_{V}$ mit entsprechenden Randbedingungen eines Sensors ist in Bild 2 gezeigt. Die Einsatzschranken sind dabei von den Durchmessern der beiden Düsen abhängig. In den 60er bis 80er Jahren wurden eingehende Untersuchungen pneumatischer Abstandssensoren durchgeführt (vgl. [5], [6]). Heute sind aufgrund großer Fortschritte in der Leistungsfähigkeit von Rechnersystemen neue Betrachtungen (z.B. numerische Berechnungen wie Strömungssimulation / Computational Fluid Dynamics (CFD)) möglich. Hierzu wurden erste Forschungsergebnisse von den Autoren veröffentlicht (vgl. [4], [7]).

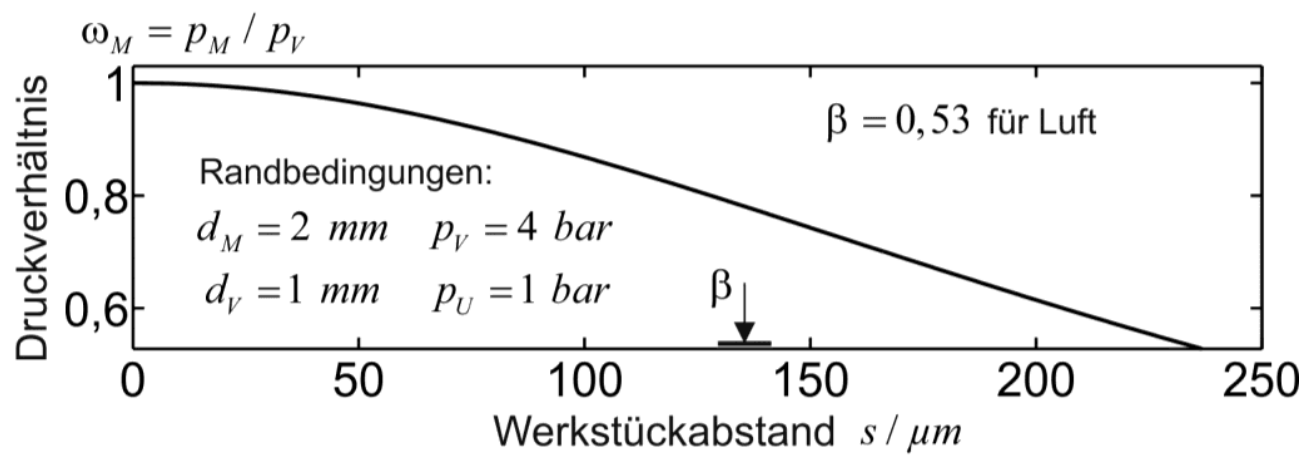

Bild 2: Beispiel-Kennlinie pneumatischer Abstandssensoren

\section{Ansätze zur virtuellen pneumatischen Abtastung}

Um den Sensor (Bild 1) zu modellieren gibt es verschiedene Ansätze. Vorgestellt werden eine geometrische Betrachtung sowie die numerische Strömungsmechanik (CFD). Grundlagen wie das dynamische Verhalten wurden vorab untersucht [7].

\subsection{Geometrische Modellierung}

Ein Ansatz zur virtuellen Abtastung ist die Beschreibung der Strömung über ihren engsten Querschnitt $A_{M}$. Aufgrund von Unebenheiten auf der Oberfläche wie Rauheit oder Formabweichungen ist der Ringspalt zwischen Messdüse und Messobjekt kein idealer Zylindermantel. Wird aber diese Mantelfläche unter Berücksichtigung der wirklichen Oberfläche des Messobjektes berechnet, kann die geometrische Filterwirkung beschrieben werden. Dieses Modell ist in Bild 3a)-b) dargestellt. Ein Profil $(x, z)$ wird mit dem Abstand $\Delta x$ und die Messdüse mit 
dem Radius $r$ in Winkelsegmente der Breite $\Delta \varphi$ diskretisiert a). Auf Basis dieser Unterteilung werden $(x, z)$-Wertepaare, die auf den diskretisierten Punkten der Messdüse liegen, interpoliert $\left(x_{i p}, z_{i p}\right)$ b), indem die Punkte der Messdüse auf die Oberfläche des hier anisotropen Messobjektes projiziert werden. Der gemittelte Wert $\bar{s}$ des Abtastpunktes $i$ kann durch Integration entlang der Mantelfläche berechnet werden. Die kontinuierliche Darstellung lautet mit der Position des Messdüsenausgangs $\left(x_{0, i}, z_{0}\right)$ :

$$
\bar{s}_{i}=\frac{1}{2 \pi} \int_{0}^{2 \pi}\left(z_{0}-z_{i p}\left(x_{0, i}+r \cdot \cos (\varphi)\right)\right) d \varphi .
$$

Die zur numerischen Implementierung nötige Überführung in $N$ gleich große Abschnitte lautet:

$$
\bar{s}_{i} \approx \sum_{j=1}^{N} \frac{\left(z_{0}-z_{i p}\left(x_{0, i}+r \cdot \cos (\mathrm{j} \cdot \Delta \varphi)\right)\right.}{N} .
$$

Die Fläche des engsten Querschnitts berechnet sich somit zu:

$$
\bar{A}_{M}(i)=\bar{s}(i) \cdot \pi \cdot d_{m} .
$$

Für $\bar{s}$ wird der zugehörige Wert des Druckquotienten $p_{M} / p_{V}$ aus der dimensionslosen Kennlinie ausgelesen. Die Berechnung wird für alle Abtastpunkte $i$ ausgeführt. Somit kann die statische Filterwirkung durch eine Mittelung der Ringspaltfläche zwischen Messdüse und Messobjekt charakterisiert werden. Mithilfe einer weiteren Integration können auch 3D-strukturierte Oberflächen abgetastet werden. Deutlich rechenaufwändiger ist das Aufstellen eines CFD-Modells. Ein Schnitt durch das Modell des Sensors am Messdüsenausgang ist in Bild 3c) dargestellt. Dieser numerische Ansatz wird nachfolgend vorgestellt.
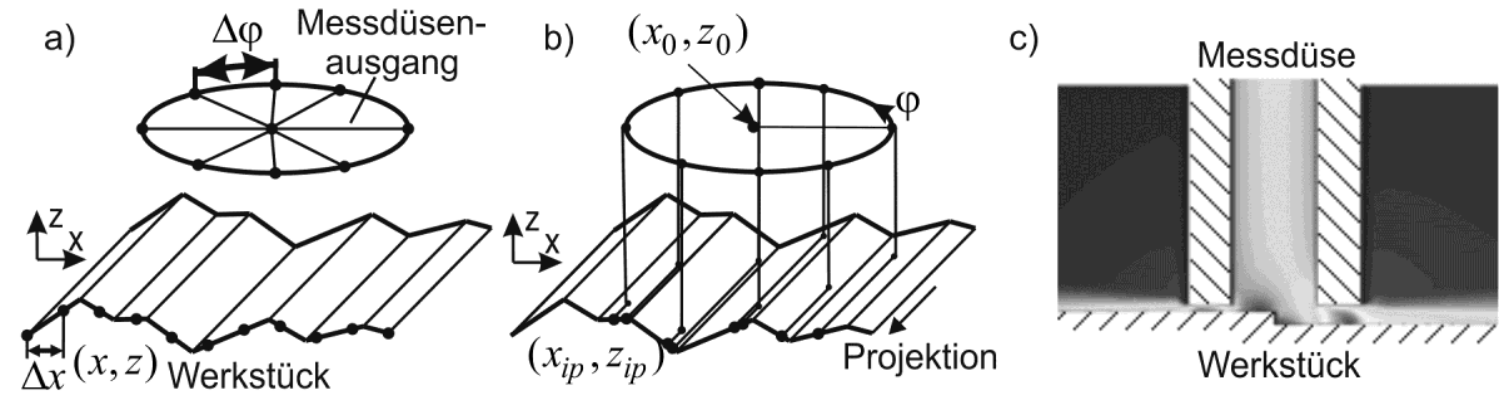

Bild 3: a) b) Vorgehen geometrische Modellierung, c) Geschwindigkeitsfeld CFD 


\subsection{Numerische Strömungsmechanik}

Der zweite Ansatz ist ein CFD-Modell, vgl. Bild 3c). Hierbei werden die strömungsmechanischen Grundgleichungen in einem diskreten Raum gelöst. Die Software ANSYS ICEM sowie ANSYS Fluent wurde hierfür verwendet. Für Grundlagen bezüglich der Methodik von CFD sei auf weiterführende Literatur [8] [9] verwiesen.

Ein 3D-Modell des pneumatischen Sensors wurde aufgebaut, das über eine Schnittstelle zur Ankopplung von Oberflächen verfügt. Für jede virtuelle Abtastung muss lediglich die Oberfläche neu vernetzt werden. Diese wird dynamisch am Sensor vorbeigeführt und der Messdruck zeitabhängig ausgelesen.

\section{Entwicklung eines Normals für pneumatische Sensoren}

\subsection{Auslegung der Geometrie}

Das Normal sollte einen Absatz zur Untersuchung aller Ortsfrequenzen bereitstellen, die Sensor-Kennlinie kalibrieren und das Filterverhalten mit Geometrieelementen spezieller Ortsfrequenzen charakterisieren. Der Prototyp (Bild 4a) wurde als Außendrehprofil auf ein Werkstück aufgebracht. Der notwendige Radius zur Vernachlässigung von Krümmungseffekten wurde mit CFD-Modellen ermittelt.

Das Normal wurde weiterentwickelt, um eine Absolut-Messung zu ermöglichen. Zur Minimierung der messkraftbedingten Verschiebung der Messeinrichtung ist eine Positionierkante auf dem Normal für die Messdüse sinnvoll. Eine Referenzebene zur Ausrichtung sowie ein größerer Messbereich werden vorgesehen sowie die Amplitude der Geometrieelemente variiert. Vorbereitend wurden Abtastungen mit geometrischer Filterung und CFD untersucht. Die Weiterentwicklung ist in Bild $4 \mathrm{~b}$ gezeigt und wurde aus Aluminium mit einem Plandrehprozess gefertigt.

Bei der taktilen Referenzmessung beider Geometrien (Bild $4 \mathrm{c}$-d) ist die kinematische Rauheit des Drehprozesses erkennbar. Ebenfalls sind Unterschiede zwischen dem Außendreh- und Plandrehprozess feststellbar. So weist der Plandrehprozess eine höhere Geradheitsabweichung, jedoch auch eine geringere kinematische Rauheit bedingt durch die Werkzeug-Geometrie auf. 
a) Prototyp

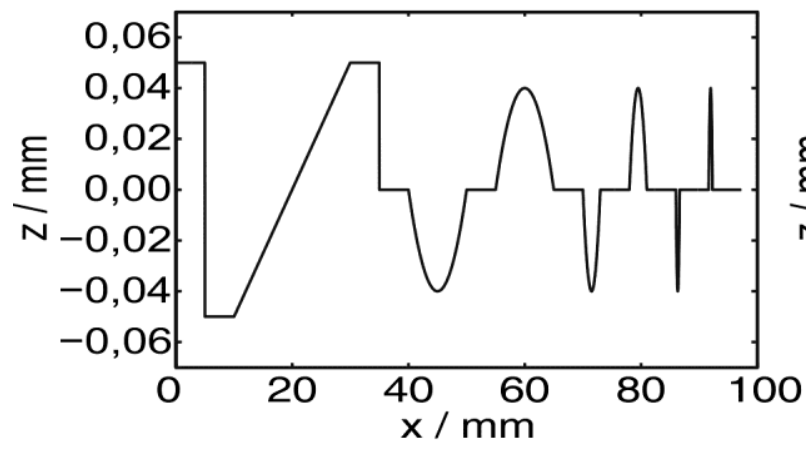

c) Prototyp Referenz

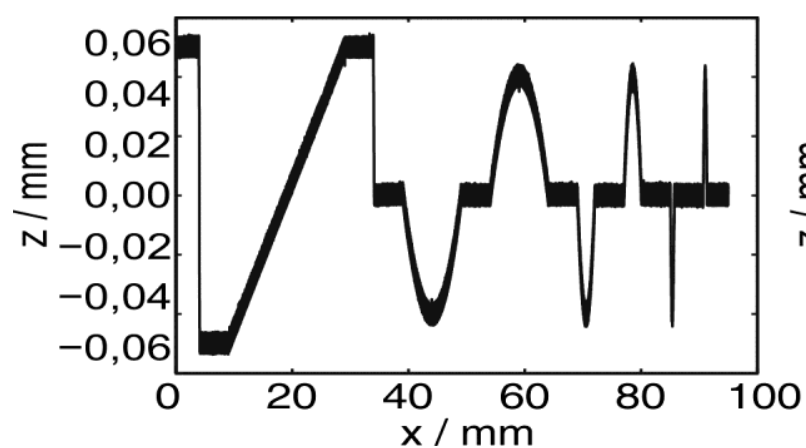

b) Weiterentwicklung

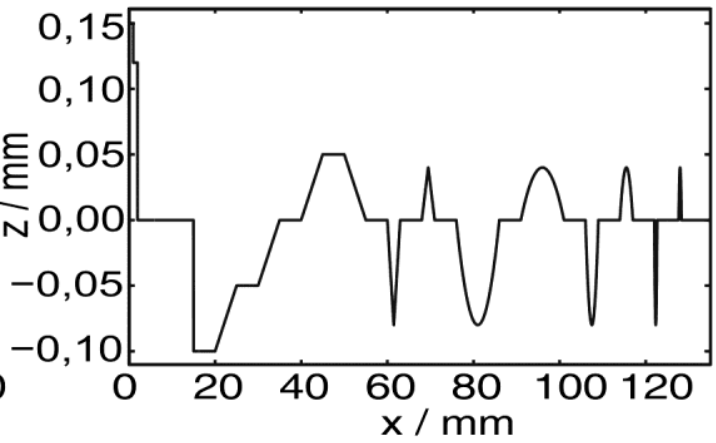

d) Weiterentwicklung Referenz

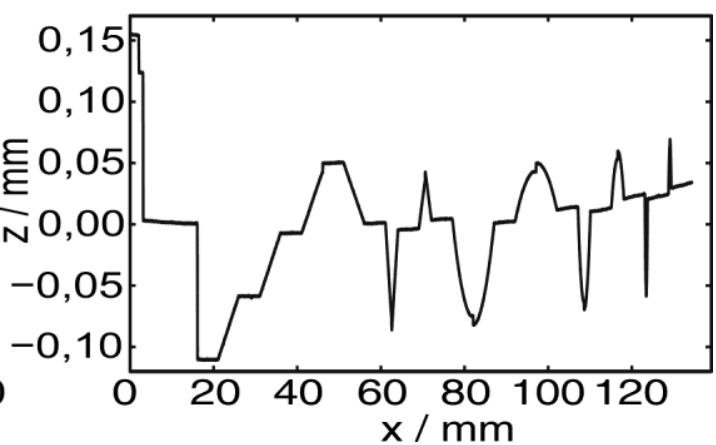

Bild 4: Normal, Geometrie Prototyp und Weiterentwicklung, Soll- und Ist-Daten

\subsection{Abtastung}

Die pneumatische Abtastung wurde auf verschiedene Weise durchgeführt:

- mit geometrischer Filterung der Soll- und Referenzdaten (Bild 5 i)

- mit Abtastung der Soll-Daten mit CFD (Bild 5 ii)

- sowie als pneumatische Abtastung im Experiment (Bild 5 iii).

Grau dargestellt sind die Ideal-Geometrie sowie für Bild 5bi) und biii) die Referenzgeometrie, um Glättungseffekte bei hohen Ortsfrequenzen zu verdeutlichen. Die Abtastungen in CFD sowie im Experiment wurden langsam ausgeführt, um dynamische Effekte zu vernachlässigen. Die CFD-Berechnung wurde zur Verkürzung der Rechenzeit auf zwei Simulationen aufgeteilt. Auch die Datensätze der Messung sind aus zwei Abschnitten fusioniert. Die Umrechnung von Druck auf Abstand erfolgt mit der in der jeweiligen Messung kalibrierten Kennlinie. Dabei werden der gefilterte mittlere Werkstückabstand und das dimensionslose Druckverhältnis korreliert. Beim Experiment wird das Normal mit einer Linearachse verfahren. Der Sensor wird an die Positionierkante angelegt und der Kontakt mit 
einem Stromkreis überprüft. Dann wird der Sensor wieder vom Werkstück entfernt und die Verschiebung mit einem kapazitiven Sensor referenzgemessen. So kann eine absolute Positionierung (ca. $\pm 3 \mu \mathrm{m}$ ) und pneumatische Messung erfolgen. Dieser Prozess könnte als Kalibrierstrategie automatisiert werden. Das Werkstück wird eingespannt, an der Referenzfläche ausgerichtet und der Kontakt eingestellt. Aus der Abtastung ergibt sich eine mögliche Justierung der Kennlinie.

Prototyp

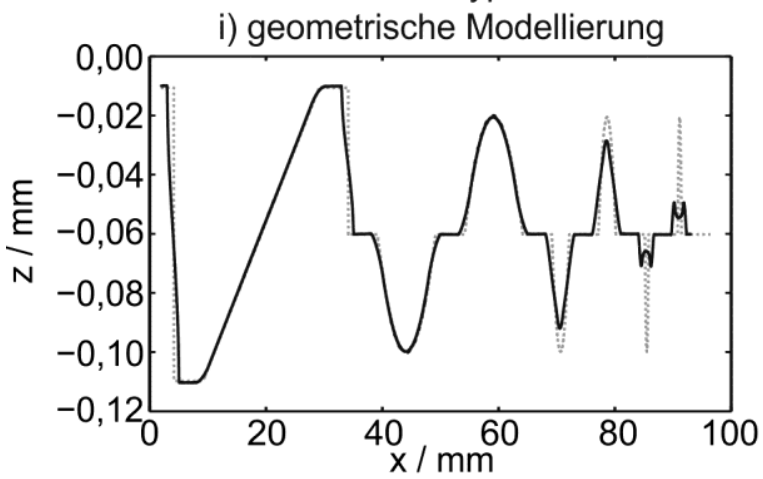

ii) CFD-Simulation

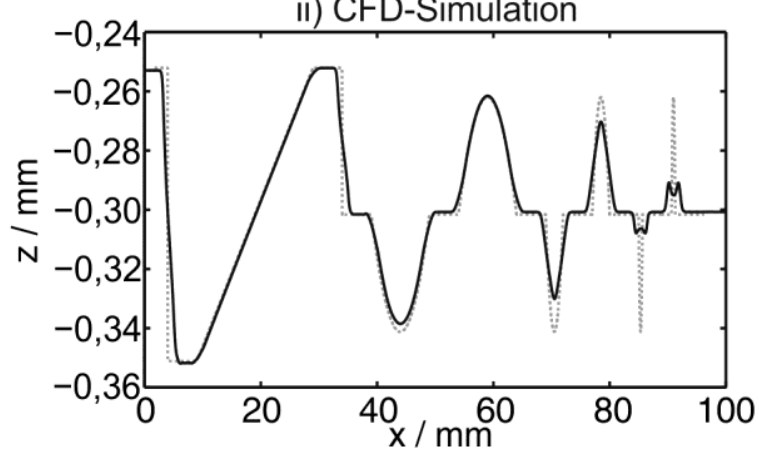

iii) Experiment

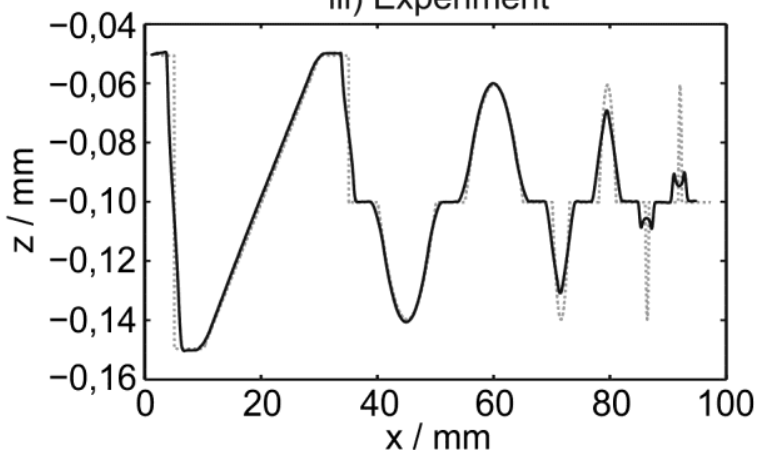

Weiterentwicklung

i) geometrische Modellierung

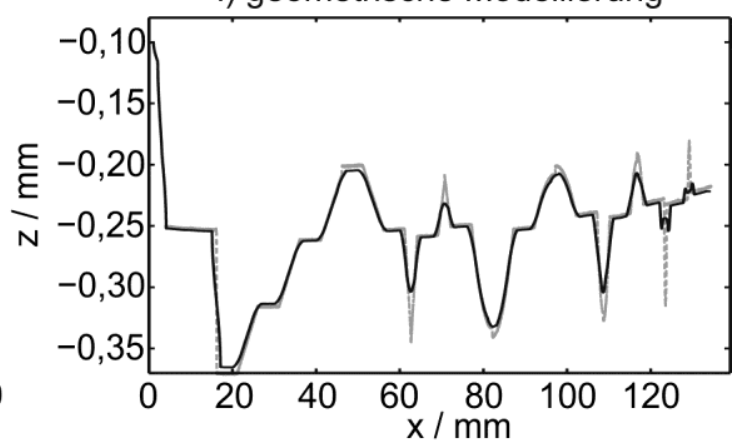

ii) CFD-Simulation

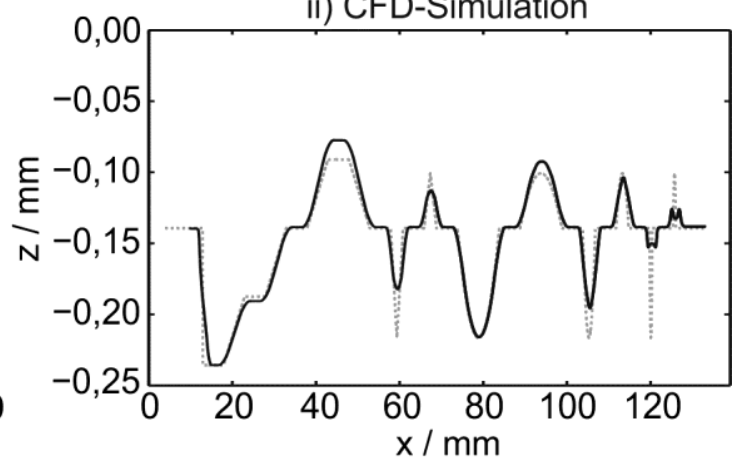

iii) Experiment

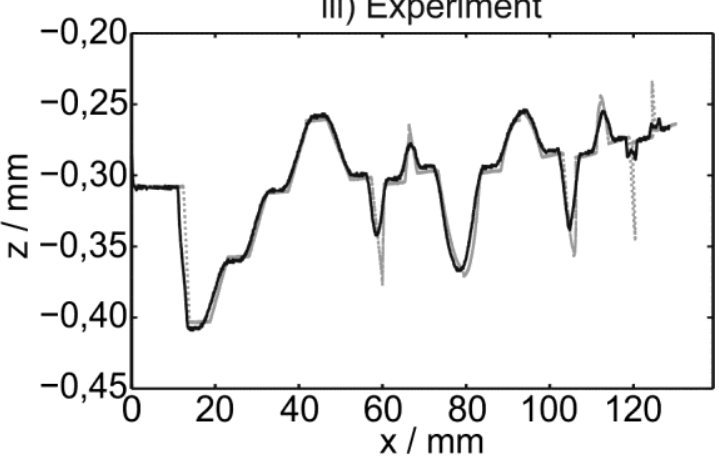

Bild 5: Pneumatik-Normal: Überblick über die Abtastergebnisse

Beim Vergleich der Abtastergebnisse werden das CFD-Modell mit der geometrischen Filterung der Soll-Daten, sowie die Messung mit der geometrischen Filterung der Referenzdaten verglichen. Die Darstellung der Abweichungen ist in Bild 6 gegeben. Es kann festgestellt werden, dass der Glättungseffekt durch die Mess- 
düse in der CFD-Simulation und der Messung aufgrund von Reibungseffekten etwas stärker ausgeprägt ist. Das sehr einfache Modell der geometrischen Modellierung stellt jedoch schon eine gute Näherung des Abtastverhaltens sowohl von Experiment als auch von CFD-Simulation dar. Es kann somit gezeigt werden, dass mit sehr geringem Rechenaufwand der komplexe Zusammenhang der Strömung durch einen pneumatischen Sensor mit nur geringfügigen Abweichungen dargestellt werden kann.

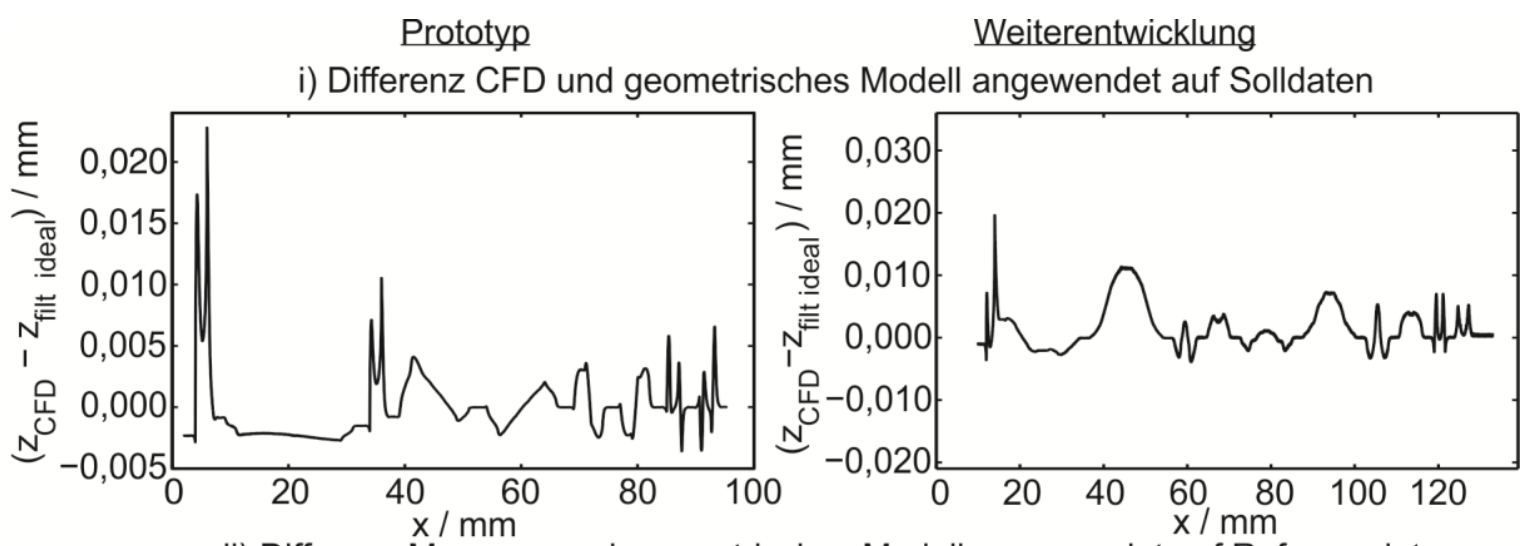

ii) Differenz Messung und geometrisches Modell angewendet auf Referenzdaten
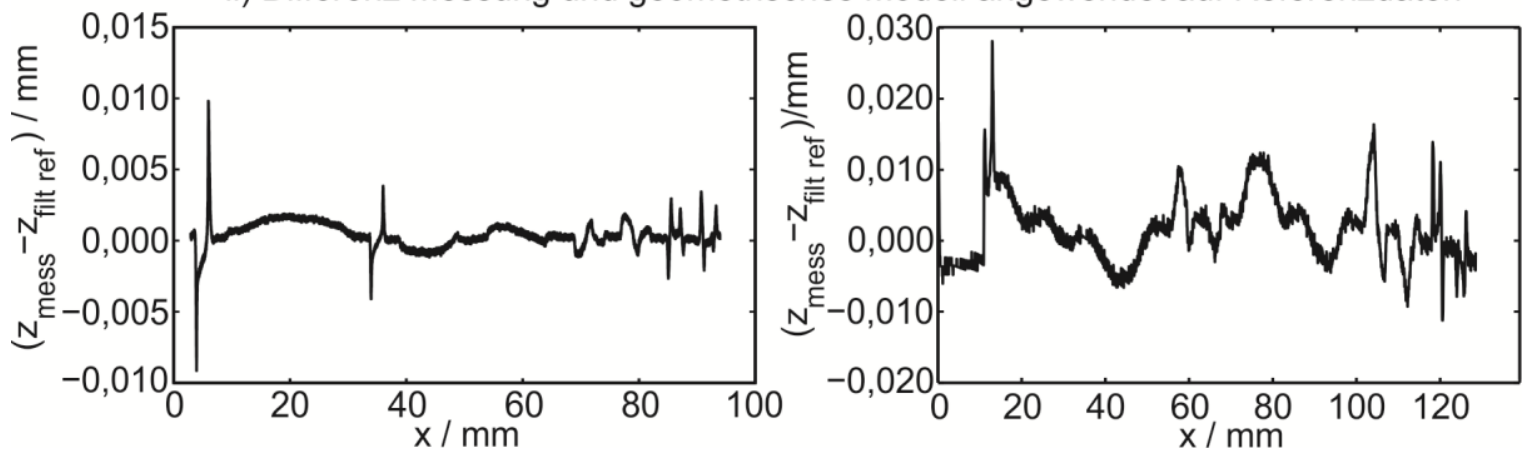

Bild 6: Vergleich der Abtastungsergebnisse

\section{Zusammenfassung}

Ein neues Normal für die Kalibrierung pneumatischer Abstandssensoren wurde vorgestellt. Die Auslegung der Geometrie erfolgte anhand virtueller Abtastungen. Methoden zur Modellierung pneumatischer Abtastungen wurden vorgestellt und funktionieren zuverlässig. Ansätze einer Kalibrierstrategie sind erarbeitet worden. Zwischen dem vorgestellten Modell zur geometrischen Filterung und den Experimenten sowie den rechenaufwendigeren CFD-Simulationen konnte eine gute Übereinstimmung der Abtastergebnisse erzielt werden. 


\section{Danksagung}

Das Projekt wird vom europäischen Fonds für regionale Entwicklung (EFRE), der Jenoptik Industrial Metrology Germany GmbH und der FZH Feinmechanische Zerspanungs-GmbH unterstützt, wofür wir uns recht herzlich bedanken möchten.

\section{Literatur}

[1] BIPM: International vocabulary of metrology - Basic and general concepts and associated terms (VIM), in JCGM 200, 2008

[2] Trumpold, H.: Längenprüftechnik - eine Einführung, 2. Auflage, VEB Fachbuchverlag, Leipzig, 1984

[3] Lemke, E.: Fertigungsmesstechnik, Friedr. Vieweg \& Sohn Verlagsgesellschaft $\mathrm{mbH}$, Braunschweig, 1988

[4] Schäfer, P.; Eifler, M.; Seewig, J.; Volk, R.: Pneumatische Abstandsmessung, Funktionen und potentielle Einsatzgebiete, Tagungsbald VDI Fachtagung Form- und Konturmesstechnik, Leonberg, 04.-05. Juni 2013

[5] Wiemer, A.: Pneumatische Längenmessung, VEB-Verlag Technik, Berlin, 1970

[6] Schulz, H.: Die Pneumatik in der Längenmesstechnik, Hanser-Verlag, München, 1967

[7] Schäfer, P.; Eifler, M.; Seewig, J.: Pneumatische Abstandsmessung, Mathematische Beschreibung von Dynamik und Übertragungsverhalten, wt Werkstatttechnik online, S. 904-910, 11/12-2013

[8] Laurien, E.; Oertel, J.: Numerische Strömungsmechanik, 5. Auflage, Springer Fachmedien Wiesbaden, Wiesbaden, 2013

[9] Ferziger, J.H.; Peric, M.: Numerische Strömungsmechanik, Springer-Verlag, Berlin, Heidelberg, 2008 